\title{
Nine-year-long complex humeral nonunion salvaged by distraction osteogenesis technique: a case report and review of the literature
}

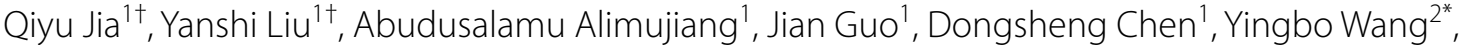 \\ Aihemaitijiang Yusufu ${ }^{1 *}$ and Chuang $\mathrm{Ma}^{1 *}$ (D)
}

\begin{abstract}
Background: Humeral nonunion with significant bone loss or shortening is uncommon and poses a complex clinical problem. We present a case of humeral nonunion with a large segmental bone defect treated with the distraction osteogenesis technique and remedy the radial nerve palsy produced during distraction osteogenesis by forearm tendon transfers. The reconstruction of upper limb function was achieved with satisfactory results. This case provides a referenceable alternative method for repairing large segmental bone defects due to complex nonunion of the upper extremity, as well as a remedy in the unfortunate event of radial nerve palsy, providing a reference and lessons learned for the treatment of similar cases and the management of possible complications.
\end{abstract}

Case presentation: A 31-year-old male patient experienced 9 years of hypertrophic nonunion due to an unreliable internal fixation. The radiographs showed the absence of bone bridging between the two fragments, loosening of the screws, and extensive osteolysis around the internal screws. The patient was treated with distraction osteogenesis. At the end of the distraction period, the patient unfortunately developed right radial nerve paresis, which was salvaged by forearm tendon transplantation, and finally reconstructed hand function and achieved bone union of the humerus.

Conclusion: Distraction osteogenesis, although not a panacea for all humeral nonunions with significant segmental bone loss, does offer a viable salvage procedure in this unusual and often complex clinical problem. When irreversible radial nerve palsy occurs during distraction, forearm tendon transfers can have a good clinical effect.

Keywords: Humeral nonunion, Distraction osteogenesis, Radial nerve palsy, Tendon transfers, Case report

\section{Background}

Humeral nonunion is estimated to account for 2 to $10 \%$ of surgically treated patients [1-3]. Nonunion humeral shaft fractures often lead to pain, prolonged disability leading to reoperation, long-term absence from work,

\footnotetext{
*Correspondence: ybwang20002575@163.com; ahmatjang@163.com; 1516175919@qq.com

${ }^{\dagger}$ Qiyu Jia and Yanshi Liu contribute equally to this study

${ }^{1}$ Department of Microrepair and Reconstruction, The First Affiliated

Hospital of Xinjiang Medical University, Urumqi, Xinjiang, China

${ }^{2}$ College of Chemical Engineering, Xinjiang Normal University, Urumqi,

Xinjiang, China
}

and impaired quality of life. An increased incidence of nonunion is associated with many factors, including smoking, abusing alcohol, using anti-inflammatory drugs, unstable fixation, poor patient compliance, devitalization of soft tissues, insufficient immobilization, and infection $[4,5]$.

Reconstruction of humeral shaft nonunions is a challenge for orthopedic surgeons, especially for osteoporosis, bone defects, and infection [6,7]. Several strategies have been proposed to resolve these complex problems, including (double) plating augmented with autologous 
bone grafts, locking intramedullary nails, unilateral external fixators, and circular external fixation [8-12].

A patient with distal humerus nonunion for nine years is described in the present study. A plate was implanted for internal fixation as preliminary management. Unfortunately, unstable fixation led to hypertrophic nonunion, resulting in bone resorption around the screws and a large bone defect. Ultimately, the injured extremity was salvaged successfully by distraction osteogenesis, forearm tendon transfers remedied a series of symptoms of radial nerve palsy caused by distraction osteogenesis, and satisfactory clinical outcomes were finally achieved.

\section{Case presentation}

A 31-year-old man presented with nonunion of the right humeral diaphysis. He sustained a closed fracture nine years ago due to a fall from a height and was treated with internal fixation at a local hospital. The patient's condition improved, was discharged and did not return to the hospital for a clinical follow-up. Two years ago, the patient suddenly had abnormal motion in the right upper arm, accompanied by pain, while performing daily living activities. The patient did not go to the hospital for examination and treatment due to personal reasons. The pain symptoms were gradually relieved after using over-the-counter pain relievers. However, the patient continued to experience abnormal activity of the right upper arm, and experienced obvious pain during strenuous activities. Twenty days ago, the patient developed active protrusions on the inner skin of the right upper arm, accompanied by obvious pain, and came to our department for help. The patient complained of moderate pain at the nonunion site that worsened with stress, in addition to pathological barriers to motion and severe functional disability of the injured limb. The radiographs showed the absence of bone bridging between the two fragments, loosening of the screws, and extensive osteolysis around the internal screws (Fig. 1a). The diagnosis was considered to be humeral nonunion.
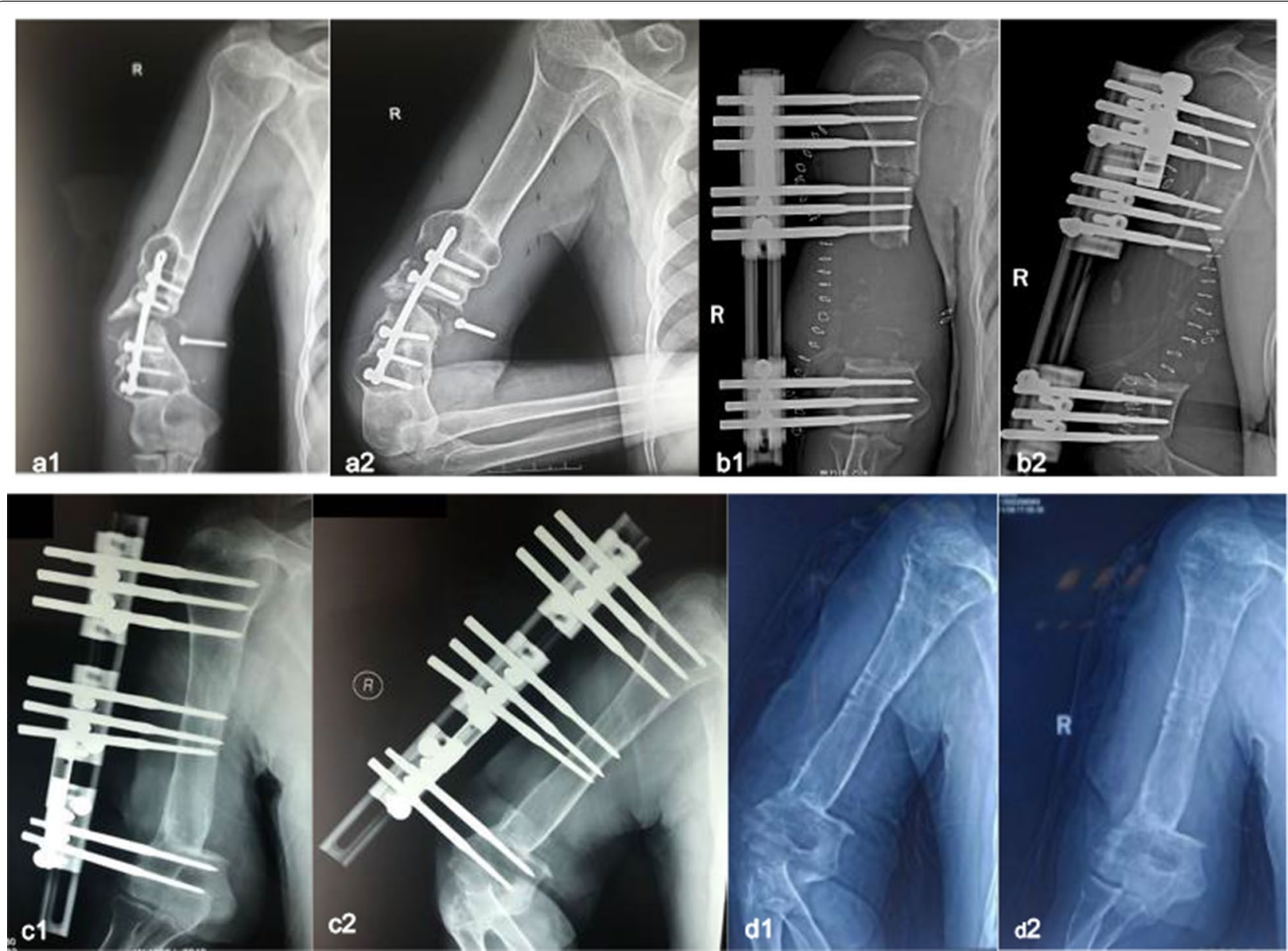

Fig. 1 Radiographs of this patient at different stages of distraction osteogenesis treatment. a1, a2 Preoperative. b1, b2 Postoperative. c1, c2 8 months postoperatively. $\mathbf{d} \mathbf{1}, \mathbf{d} \mathbf{2} 12$ months after removal of the external fixator 
The operation was performed under general anesthesia. Serious scar adhesion and chaotic local anatomy were observed after a full-length incision on the lateral side of the right humerus. We separated the subcutaneous tissue and explored the radial nerve, which was dark in color and complete in continuity. The radial nerve was carefully separated from the proximal end to the distal end. It was wrapped by abnormal hyperplasia of bone $5 \mathrm{~cm}$ above the lateral condyle of the humerus, and there was local compression (Fig. 2a1). The hypertrophic bone callus was carefully excised using a rongeur, and the radial nerve was released and protected by rubber strip traction. When the periosteum was separated to depth, a large amount of callus was observed between the fracture gaps, but the cortical bone was uneven (Fig. 2a2). Part of the cortical bone was removed, the plate was found to be wrapped by the bone of the hollow structure, and the screw and plate were loosened. The bone around the implant was removed, and the plate and screw were removed. The distal humerus and the elbow joint cavity were connected. The bones in the affected area of the humerus were all sclerotic bones, and there was no medullary cavity or poor blood supply. Radical debridement was performed to reopen the intramedullary canal, including bone decortication, excision of fibrous tissue and ablation of necrotic bone. Under the image intensifier, 8-9 parallel pins were inserted at the lateral side of the humerus, perpendicular to the anatomical axis. Three pins were fixed at the proximal and distal ends of the humerus. The other three pins were inserted into the transported bone segment. All pins were confirmed to be parallel to each other in the same plane. A minimally invasive subperiosteal osteotomy was performed between the two sets of pins at the proximal end of the humerus (Fig. 2a3, b).

The lengthening procedure was initiated on the seventh postoperative day. Based on the patient's pain tolerance, a 120-day lengthening period allowed for $8.5-\mathrm{cm}$ lengthening (Fig. 1b), a rate of $0.71 \mathrm{~mm} /$ day followed by eight months of a consolidation phase (Fig. 1c). Eventually, the bone completely healed. The external fixation index (EFI) was 43.18 days $/ \mathrm{cm}$ for this patient.

This patient presented with radial nerve palsy with a wrist drop and obstructed thumb and finger extension 135 days after the surgery ( 1 week after the distraction phase was completed at the first clinical visit) (Fig. 3a2). Additional surgical interventions were performed to manage radial nerve palsy.
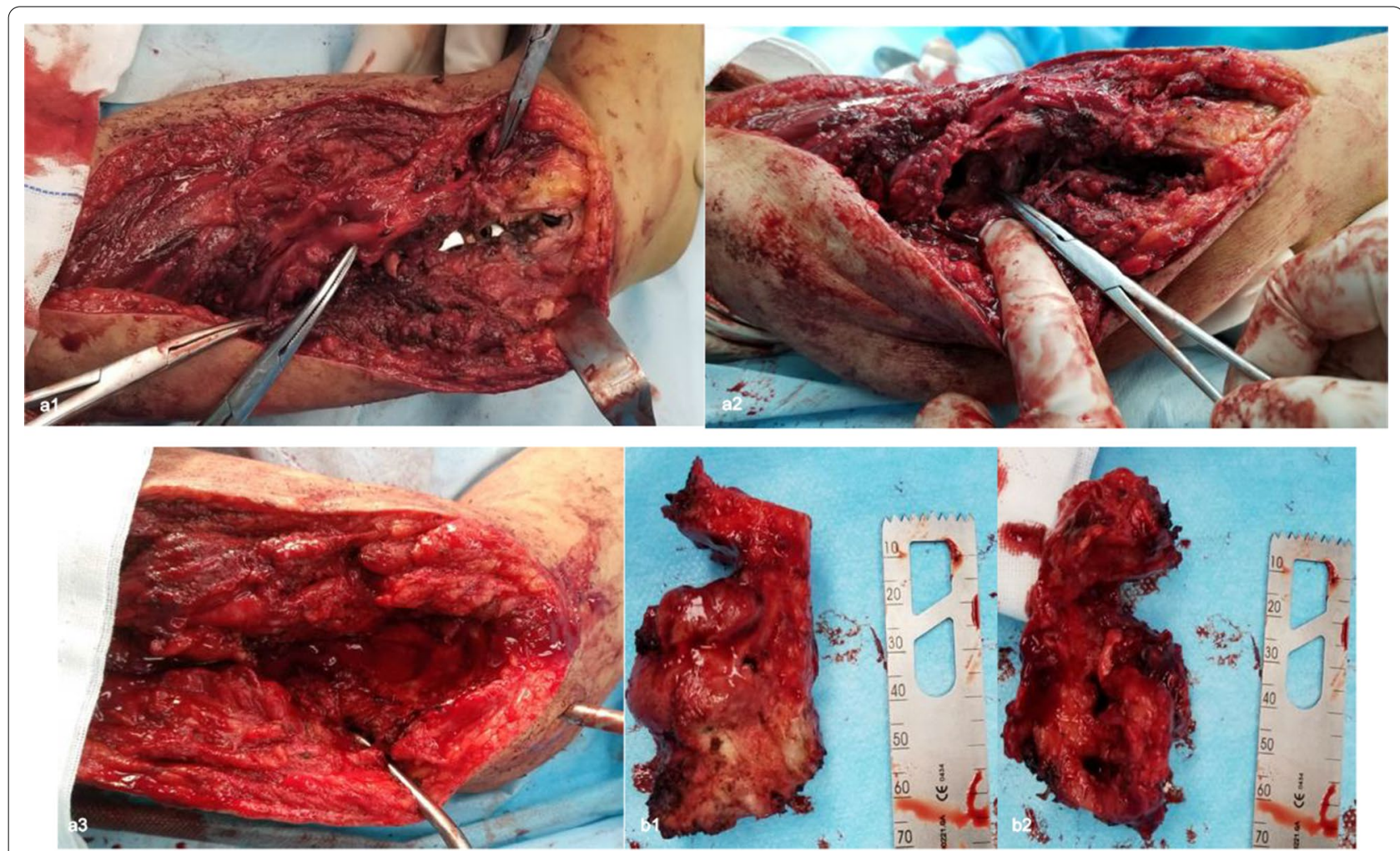

Fig. 2 Intraoperative pictures of the affected area. a1 Hypertrophied bone encapsulating the radial nerve. a2 Disorganized bone structure. a3 Bone defect area after amputation of abnormal hypertrophy. $\mathbf{b}$ Hypertrophic bones $\mathbf{b} \mathbf{1}$ and $\mathbf{b} \mathbf{2}$ were removed intraoperatively 


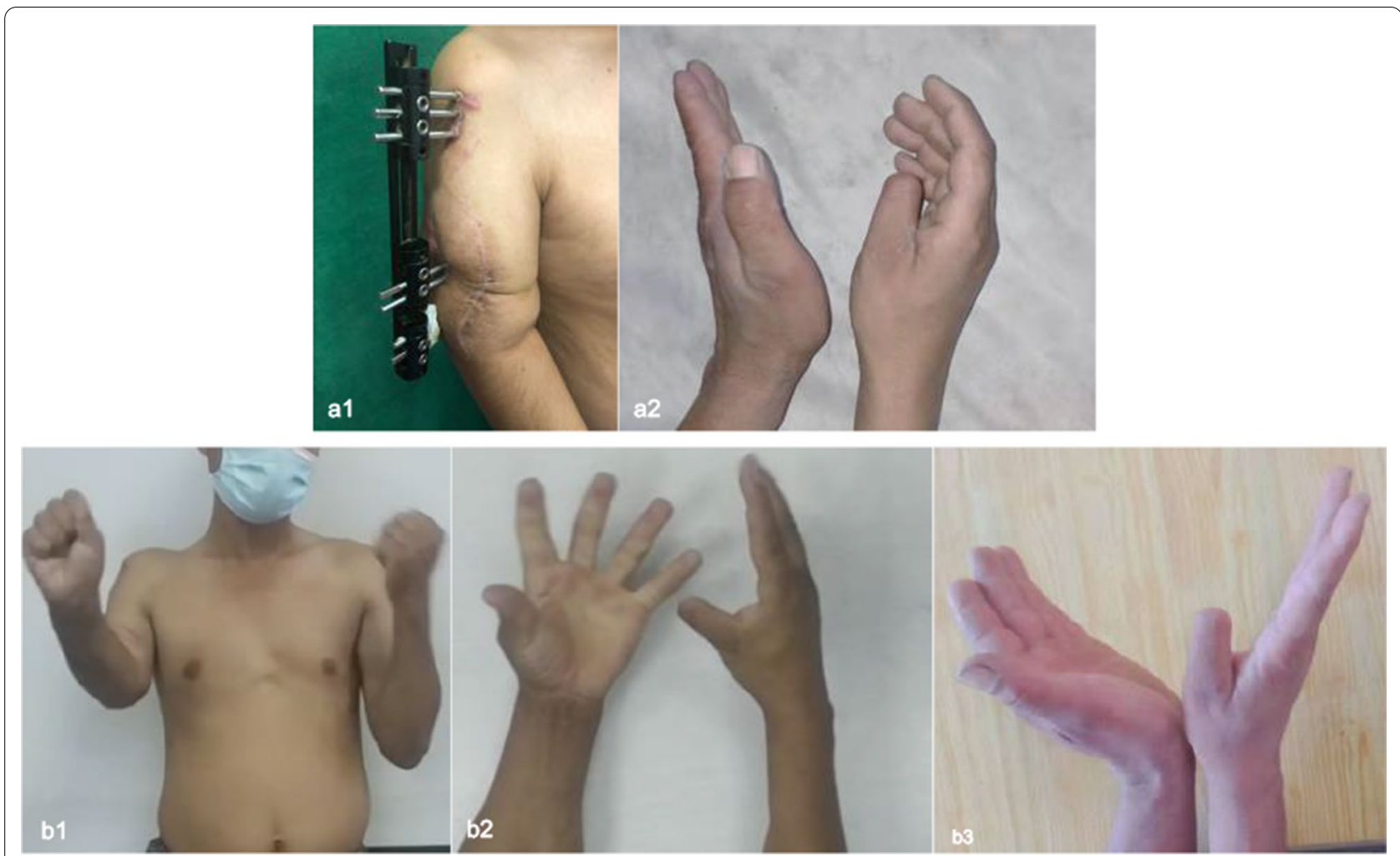

Fig. 3 The patient showed radial nerve paresis at the end of distraction osteogenesis. a1 Muscle buildup around the distal humerus at the end of distraction. a2 The right hand showed wrist extension, finger extension, and thumb extension dysfunction. b1, b2 Radial nerve function was reconstructed after forearm tendon transfers

A lateral incision of the right upper arm was made to delicately separate the tissue layer-by-layer under the microscope, avoiding the brachial artery. The brachial artery was buried in the scar tissue and then completely released. Serious incarceration and degeneration of the radial nerve caused by the surrounding soft tissues at the lengthening of the lower humerus could be observed. The proximal radial nerve lost its normal shape in a large area. Neural anastomosis and transplantation could not be performed during intraoperative evaluation, and right forearm tendon transplantation was therefore conducted to reconstruct back extension function.

A longitudinal incision was made at the junction of the middle and upper third of the radial side of the right forearm and entered from between the brachioradialis and the extensor carpi radialis longus to locate and cut the pronator teres (PT), displace and anastomose it to the extensor carpi radialis brevis (ECRB). Next, the flexor carpi ulnaris (FCU) was found and cut, in which the FCU was anastomosed to the extensor digitorum cornmunis (EDC) around the ulnar border of the forearm. At the same time, the extensor pollicis longus (EPL) was severed at the musculotendinous junction, drawn out at the metacarpophalangeal joint of the thumb, and rerouted to the palmaris longus (PL) through the tunnel of the abductor pollicis longus.

After docking, the patient was encouraged to perform progressive functional exercises. One month after the end of the consolidation period, sufficient consolidation in the distraction zone and complete union at the docking site was determined, and the external fixator was removed. At the same time, the patient had fair wrist extension activity, grade 3 muscle strength, and grade 4 muscle strength for flexion.

At the last clinical visit (12 months after frame removal), the patient had good wrist extension activity, good thumb extension and finger extension function (Fig. 3b). Elbow range of motion was slightly limited $\left(10^{\circ}\right.$ to $90^{\circ}$ ), but based on the Mayo criteria, was subclassified as good, and hand grip strength increased without significant difficulty in performing daily living activities.

\section{Discussion and conclusions}

Humeral nonunion with significant bone loss or shortening is an uncommon and complex clinical problem [13]. The etiology includes bone loss due to open fractures, infection, tumor resection, and bone resorption associated with failed internal fixation [13-15]. Although the 
methods to address humeral nonunion have significantly improved, the surgeon performing the procedure will experience challenges in the clinical setting regarding occasional complex cases.

Achieving biomechanical stability is a reasonable method for treating nonunion humeral fractures. Compression plates, which are now widely used, are not always sufficiently stable unless the soft tissue surrounding the fracture is widely incised, especially when osteopenia is present or previous attempts at fixation have failed. In addition, this method is not without complications, the most important of which is damage to the radial nerve (3 to 29\%) [16], as at least six cortical screws are fixed on either side of the nonunion $[8,14]$. Reconstruction with two plates at right angles has been advocated to increase local stability, but there were no significant differences in clinical outcomes between single- and double-plate constructions [17]. This technique is not recommended for nonunions with infection, osteoporotic bone, long spiral, or large segmented fracture lines, especially distal metaphyseal nonunions, as more than six cortices or augmentation with strut grafts are required [16]. In this case, nonunion of the distal humerus was not suitable for acute shortening due to the large bone defect area, and there was insufficient space to engage six cortices. If it was decided that osteotomy would not be performed, it was clear that the hollow structure of the hypertrophied bone would not provide favorable support for the plate.

Intramedullary locking nails are controversial in the treatment of humeral nonunions [18]. Unlike lower limb nonunion, lack of weight bearing status and inadequate compression diminish the success rate in humeral shaft nonunions [19]. Autologous bone grafting is osteogenic, osteoinductive, and osteoconductive. However, the traditional supply of autogenous materials may be insufficient when the bone defect in the nonunion area is large. Fibula transplantation is also a good choice when there are segmental defects in humeral nonunion. Heitmann et al. described 15 patients with segmental defects who were treated with an osteoseptocutaneous fibular transplant and showed good functional and cosmetic results. This technique is, however, complex and associated with a high rate of complications.

External fixation conserves the soft-tissue envelope and the vitality of the remaining bone [8]. This technique is less invasive and can minimize surgical damage to the fracture. The fixator provides gradual compression to the nonunion site, mimicking the weight-bearing status of the lower limbs [20]. In addition, in the process of fixation, the external fixator can evenly distribute the force on the bone section; effectively eliminate the shear, torsion, and displacement of the fracture end that affects the healing of the fracture; and can adjust the pressure after the operation to achieve lengthening and compression at the same time.

As mentioned earlier, this patient had extensive osteolysis around the implant plate and abnormal callus hyperplasia caused by unstable internal fixation. Bone is a mechanosensitive organ that can respond to the introduction of mechanical stimuli. When high strain forces are present at the fracture site, i.e., inadequate stability, fibrous tissue will remain, and a stable bony callus cannot form. If this condition persists for a long period of time, fibrous nonunion will develop [21]. To date, many mechanical factors that influence fracture healing have been identified, including the magnitude and direction of interfragmentary movement (IFM), the type of fracture, and the geometry of the fracture [22-24]. Notably, when the IFM exceeds a critical level, the vessels are repeatedly disrupted and unable to form at the fracture site, thus preventing the development of stable tissue [25]. A certain amount of mechanical instability can lead to greater IFM and thus increase the risk of nonunion. Severe bone resorption was present in this patient. Previous pathological studies revealed severe inflammatory reactions around failed orthopedic implants $[26,27]$. Unstable internal fixation inevitably causes long-term tissue damage, which leads to a continuous progression of inflammation [28]. This will eventually lead to increased osteoclast production and overactivation [29], inducing the development of bone resorption around the internal fixation. We elected to remove the original plate and remove the surrounding abnormal bone. Then, the upper and middle sections of the humerus were osteotomized, and unilateral external fixator bone lengthening was performed.

Most investigators believe that limb length discrepancy is not a major problem in the upper limb, and lengthening is only required if the discrepancy exceeds $5 \mathrm{~cm}$ [30, 31]. After removing the abnormal bone, our patient had a gap of approximately $8.5 \mathrm{~cm}$. Obviously, acute shortening was not applicable at that time and needed to be treated by means of bone lengthening. The potential for effective lengthening of the humerus was recognized. The incidence of callus formation during distraction was significantly higher than that of the tibia [32]. In the lower extremities, when the lengthening exceeds $20 \%$, the incidence of complications increases, but this does not occur in the humerus [33, 34]. Obviously, this patient was suitable for humerus lengthening.Monolateral external fixation instead of the Ilizarov frame or hybrid fixation was used due to its lower complication rate $[35,36]$. In addition, a monorail external fixator was sufficient to achieve lengthening and was more convenient to wear, with less negative impact on the patient's lifestyle [37]. 
Iatrogenic nerve injury, a complication related to limb lengthening, poses a challenge for orthopedic surgeon [38], as the nerves are forced to stretch along with other anatomical structures. A nerve might also become compromised as a result of the use of fixation devices placed near the nerve itself [39]. The incidence of peripheral nerve injury after limb lengthening procedures ranges from 3 to $30 \%$ [40]. In this case, the symptoms of radial nerve paralysis appeared after the lengthening period. Due to muscle attachment to the transitional bone, with the development of the distraction process, the muscle attached to the transitional bone moves distally together, resulting in the accumulation of muscle around the distal humerus (Fig. 3a1), compressing the radial nerve and resulting in radial nerve palsy.

Radial nerve injuries can permanently limit physical function, which is largely unacceptable to the patient. Functional reconstruction at this point is critical. Indications for radial nerve palsy with tendon grafting include severe injury where neurorrhaphy is not possible, brachial plexus injury, absence of extensor musculo-tendinous units, no clinical and electromyographic signs of recovery 6 months after neurorrhaphy, late presentation (>10 months) or severe scarring or muscular atrophy. High radial nerve palsy, as demonstrated in this patient, results in paralysis of all extensor muscles, leading to wrist drop. When the injury occurs proximal to the forearm, the Radial nerve palsy can be low. In such cases, wrist dorsiflexion is preserved due to sparing of the extensor carpi radialis longus (ECRL), but the fingers and thumb cannot be extended.

For surgical treatment, triple tendons transfer will be required for high radial nerve palsy, whereas for low radial nerve palsy, restoration of wrist extension may not be required [41]. If the radial nerve is completely unrecoverable, an end-to-end transfer should be performed, which will result in a straight direction of tension, but if recovery of the nerve is still possible, an end-to-side transfer of all tendons should be performed so that the native tendon can resume its function once recovery is achieved [42,43]. There are various combinations of tendon transfers for radial nerve palsy. The most common are the use of PT transfer to ECRB to reconstruct wrist extension and PL transfer to EPL to restore thumb extension. For reconstruction of finger extension, in addition to FCU, there are also options for Flexor carpi radialis (FCR) and flexor digitorum superficialis (FDS) to dock with EDC. All three of these common transfer modalities have achieved good results in previous clinical treatments.

We adopted forearm tendon transfers to achieve reconstruction of wrist extension, finger extension, and thumb extension and abduction and achieved good results after surgery. Intraoperatively, it is important to note that when the FCU is transferred to the EDC, the four EDC tendons should be divided distal to their musculotendinous junction and woven into the FCU. This should be done near the extensor retinaculum to maintain the smoothest possible glide and to prevent bow-stringing from occurring if the extensor retinaculum is divided. When PL is transferred to EPL, the EPL should then be removed from the third extensor compartment and transposed radially to Lister's tubercle. This allows for a straighter line of traction from the PL to the EPL. It also provides some additional radial adduction of the thumb in addition to extension. Of course, prior to this unfortunate event, it may have been wise to release the radial nerve in advance to prevent radial nerve palsy when the grafted bone did not reach the docking site during the extension period. The patient was very satisfied with the final overall surgical result and had a much-improved quality of life compared to the previous outcome.

This case demonstrates the successful treatment of a complex humeral nonunion with distraction osteogenesis, providing a referable alternative method for repairing a large segmental bone defect due to similar conditions in the upper extremity, as well as salvage measures in the unfortunate event of radial nerve palsy.

There are several limitations to this case report. First, long-term follow-up results, such as 5 and 10 years, are lacking for this patient. Second, there was no in-depth assessment of the patient's relevant social and psychological functioning indicators.

\section{Conclusions}

Distraction osteogenesis, although not a panacea for all humeral nonunions with significant segmental bone loss, does offer a viable salvage procedure in this unusual and often complex clinical problem. When irreversible radial nerve palsy occurs during distraction osteogenesis, forearm tendon transfers can have a good clinical effect.

\section{Abbreviations \\ EFI: External fixation index; IFM: Interfragmentary movement; PT: Pronator teres; ECRB: Extensor carpi radialis brevis; FCU: Flexor carpi ulnaris; EDC: Exten- sor digitorum cornmunis; EPL: Extensor pollicis longus; PL: Palmaris longus; ECRL: Extensor carpi radialis longus; FCR: Flexor carpi radialis; FDS: Flexor digitorum superficialis.}

\section{Acknowledgements}

Not applicable.

\section{Disclosure}

The authors report no proprietary or commercial interest in any product mentioned or concept discussed in this article.

\section{Authors' contributions}

QJ: Conducted the study. Collected and analyzed this case. Wrote the manuscript. YL: Designed the study, analyzed this case, and edited the manuscript. AA: Planned the project. Collected this case. JG: Collected and analyzed this 
case. DC: Edited the manuscript. YW: Edited the manuscript, reviewed the manuscript. AY: Edited the manuscript, reviewed the manuscript. CM: Planned the project. Reviewed the manuscript. All authors read and approved the final manuscript.

\section{Funding}

This study was funded by the National Natural Science Foundation of China (No. 81760397, 81560357 and 52063027). CM, AY, and YW provided the necessary financial support for the study and the revision of the manuscript.

\section{Availability of data and materials}

The datasets analyzed during the current study are available from the corresponding author on reasonable request.

\section{Declarations}

\section{Ethics approval and consent to participate}

This retrospective study was approved by the Ethics Committee of The First Affiliated Hospital of Xinjiang Medical University and carried out in accordance with the ethical standards set out in the Helsinki Declaration. Informed consent was received from all participating.

\section{Consent for publication}

Written informed consent was obtained from the patient for publication of this case report and any accompanying images. A copy of the written consent is available for review by the Editor of this journal.

\section{Competing interests}

The authors declare that they have no conflict of interest.

\section{Received: 4 August 2021 Accepted: 20 February 2022} Published online: 03 March 2022

\section{References}

1. Westrick E, Hamilton B, Toogood P, Henley B, Firoozabadi R. Humeral shaft fractures: results of operative and non-operative treatment. Int Orthop. 2017:41(2):385-95.

2. Harkin FE, Large RJ. Humeral shaft fractures: union outcomes in a large cohort. J Shoulder Elbow Surg. 2017;26(11):1881-8.

3. Peters RM, Claessen FM, Doornberg JN, Kolovich GP, Diercks RL, van den Bekerom MP. Union rate after operative treatment of humeral shaft nonunion-a systematic review. Injury. 2015:46(12):2314-24.

4. Niikura T, Lee SY, Sakai Y, Nishida K, Kuroda R, Kurosaka M. Causative factors of fracture nonunion: the proportions of mechanical, biological, patient-dependent, and patient-independent factors. J Orthop Sci. 2014;19(1):120-4.

5. Gaston MS, Simpson AH. Inhibition of fracture healing. J Bone Jt Surg Br. 2007:89(12):1553-60.

6. Bassiony AA, Almoatasem AM, Abdelhady AM, Assal MK, Fayad TA Infected non-union of the humerus after failure of surgical treatment: management using the Orthofix external fixator. Ann Acad Med Singap. 2009;38(12):1090-4

7. Leiblein M, Verboket R, Marzi I, Wagner N, Nau C. Nonunions of the humerus - treatment concepts and results of the last five years. Chin J Traumatol. 2019:22(4):187-95.

8. Padhye KP, Kulkarni VS, Kulkarni GS, Kulkarni MG, Kulkarni S, Kulkarni R, Patil MD, Ravi PY. Plating, nailing, external fixation, and fibular strut grafting for non-union of humeral shaft fractures. J Orthop Surg (Hong Kong). 2013;21(3):327-31.

9. Kiran M, Jee R. llizarov's method for treatment of nonunion of diaphyseal fractures of the humerus. Indian J Orthop. 2010;44(4):444-7.

10. Burg A, Berenstein M, Engel J, Luria T, Salai M, Dudkiewicz I, Velkes S. Fractures of the distal humerus in elderly patients treated with a ring fixator. Int Orthop. 2011;35(1):101-6.

11. Hierholzer C, Sama D, Toro JB, Peterson M, Helfet DL. Plate fixation of ununited humeral shaft fractures: effect of type of bone graft on healing. J Bone Jt Surg Am. 2006;88(7):1442-7.
12. Wali MG, Baba AN, Latoo IA, Bhat NA, Baba OK, Sharma S. Internal fixation of shaft humerus fractures by dynamic compression plate or interlocking intramedullary nail: a prospective, randomised study. Strateg Trauma Limb Reconstr. 2014;9(3):133-40.

13. Jupiter JB, von Deck M. Ununited humeral diaphyses. J Shoulder Elbow Surg. 1998;7(6):644-53.

14. Ring D, Jupiter JB, Quintero J, Sanders RA, Marti RK. Atrophic ununited diaphyseal fractures of the humerus with a bony defect: treatment by wave-plate osteosynthesis. J Bone Jt Surg Br. 2000;82(6):867-71.

15. Patel VR, Menon DK, Pool RD, Simonis RB. Nonunion of the humerus after failure of surgical treatment. Management using the llizarov circular fixator. J Bone Jt Surg Br. 2000;82(7):977-83.

16. Barquet A, Fernandez A, Luvizio J, Masliah R. A combined therapeutic protocol for aseptic nonunion of the humeral shaft: a report of 25 cases. $J$ Trauma. 1989;29(1):95-8.

17. Rubel IF, Kloen P, Campbell D, Schwartz M, Liew A, Myers E, Helfet DL. Open reduction and internal fixation of humeral nonunions : a biomechanical and clinical study. J Bone Jt Surg Am. 2002;84(8):1315-22.

18. Martinez AA, Herrera A, Cuenca J. Good results with unreamed nail and bone grafting for humeral nonunion: a retrospective study of 21 patients. Acta Orthop Scand. 2002;73(3):273-6.

19. Lammens J, Bauduin G, Driesen R, Moens P, Stuyck J, Smet L, Fabry G. Treatment of Nonunion of the Humerus Using the Ilizarov External Fixator. Clin Orthopaed Related Res. 1998;353:223-30.

20. Dendrinos GK, Kontos S, Lyritsis E. Use of the ilizarov technique for treatment of non-union of the tibia associated with infection. J Bone Jt Surg. 1995;77(6):835-46.

21. Claes L, Augat P, Suger G, Wilke HJ. Influence of size and stability of the osteotomy gap on the success of fracture healing. J Orthop Res. 1997;15(4):577-84.

22. Augat P, Burger J, Schorlemmer S, Henke T, Peraus M, Claes L. Shear movement at the fracture site delays healing in a diaphyseal fracture model. J Orthop Res. 2010;21(6):1011-7.

23. Augat P, Margevicius K, Simon J, Wolf S, Suger G, Claes L. Local tissue properties in bone healing: influence of size and stability of the osteotomy gap. J Orthop Res. 1998;16(4):475-81.

24. Jiang D, Zhan S, Cai Q, Hu H, Jia W. Enhanced interfragmentary stability and improved clinical prognosis with use of the off-axis screw technique to treat vertical femoral neck fractures in nongeriatric patients. J Orthop Surg Res. 2021;16(1):473.

25. Glatt V, Evans CH, Tetsworth K. A concert between biology and biomechanics: the influence of the mechanical environment on bone healing. Front Physiol. 2016;7:678.

26. Witt JD, Swann M. Metal wear and tissue response in failed titanium alloy total hip replacements. J Bone Jt Surg Br. 1991;73(4):559-63.

27. Scales JT. Black staining around titanium alloy prostheses-an orthopaedic enigma. J Bone Jt Surg Br. 1991;73(4):534-6.

28. Guo X, Bai J, Ge G, Wang Z, Wang Q, Zheng K, Tao H, Zhang L, Zhang H, Wang $\mathrm{D}$, et al. Bioinspired peptide adhesion on Ti implants alleviates wear particle-induced inflammation and improves interfacial osteogenesis. J Colloid Interface Sci. 2022;605:410-24.

29. McDonald MM, Khoo WH, Ng PY, Xiao Y, Zamerli J, Thatcher P, Kyaw W, Pathmanandavel K, Grootveld AK, Moran I, et al. Osteoclasts recycle via osteomorphs during RANKL-stimulated bone resorption. Cell. 2021;184(7):1940.

30. Pugh DM, McKee MD. Advances in the management of humeral nonunion. J Am Acad Orthop Surg. 2003;11(1):48-59.

31. El-Alfy BS, Maaty M, Niazy T. Reconstruction of infected nonunion of the distal humerus by llizarov external fixator. Injury. 2021;52(6):1418-22.

32. Tanaka K, Nakamura K, Matsushita T, Horinaka S, Kusaba I, Kurokawa T. Callus formation in the humerus compared with the femur and tibia during limb lengthening. Arch Orthop Trauma Surg. 1998;117(4-5):262-4.

33. Hosny GA. Humeral lengthening and deformity correction. J Child Orthop. 2016:10(6):585-92.

34. Pawar AY, McCoy TH Jr, Fragomen AT, Rozbruch SR. Does humeral lengthening with a monolateral frame improve function? Clin Orthop Relat Res. 2013:471(1):277-83

35. Balci HI, Kocaoglu M, Sen C, Eralp L, Batibay SG, Bilsel K. Bilateral humeral lengthening in achondroplasia with unilateral external fixators: is it safe and does it improve daily life? Bone Jt J. 2015:97-B(11):1577-81 
36. Kadhim M, Holmes L, Gesheff MG, Conway JD. Treatment options for nonunion with segmental bone defects. J Orthop Trauma. 2017:31(2):111-9.

37. Yilihamu Y, Keremu A, Abulaiti A, Maimaiti X, Ren P, Yusufu A. Outcomes of post-traumatic tibial osteomyelitis treated with an Orthofix LRS versus an Ilizarov external fixator. Injury. 2017;48(7):1636-43.

38. Rozbruch SR, Fryman C, Bigman D, Adler R. Use of ultrasound in detection and treatment of nerve compromise in a case of humeral lengthening. HSS J. 2011;7(1):80-4.

39. Karabay N, Toros T, Ademoglu Y, Ada S. Ultrasonographic evaluation of the iatrogenic peripheral nerve injuries in upper extremity. Eur J Radiol. 2010;73(2):234-40.

40. Shanmuganathan SS, Harshavardhan JG, Menon G. Complications and outcomes following humerus lengthening-an illustrative: case report. J Orthop Case Rep. 2019;9(1):94-7.

41. Agarwal P, Kukrele R, Sharma D. Outcome of tendon transfer for radial nerve palsy using Flexor Carpi Radialis combination (Brands transfer). J Clin Orthop Trauma. 2020;11(4):630-6.

42. Ochi K, Horiuchi Y, Matsumura T, Morita K, Kawano Y, Horiuchi K. A modification of the palmaris longus-to-extensor pollicis longus transfer for radial nerve palsy. J Hand Surg Am. 2012:37(11):2357-61.

43. Sammer DM, Chung KC. Tendon transfers: part I. Principles of transfer and transfers for radial nerve palsy. Plast Reconstr Surg. 2009;123(5):169e-77e.

\section{Publisher's Note}

Springer Nature remains neutral with regard to jurisdictional claims in published maps and institutional affiliations.

- fast, convenient online submission

- thorough peer review by experienced researchers in your field

- rapid publication on acceptance

- support for research data, including large and complex data types

- gold Open Access which fosters wider collaboration and increased citations

- maximum visibility for your research: over $100 \mathrm{M}$ website views per year

At BMC, research is always in progress.

Learn more biomedcentral.com/submissions 